\title{
Distance adaptation depends upon plasticity in the oculomotor control system
}

\author{
SHELDON M. EBENHOLTZ and SHERRY K. FISHER \\ University of Wisconsin, Madison, Wisconsin
}

\begin{abstract}
Maintaining binocular fixation on a target at $20 \mathrm{~cm}$ in the absence of secondary cues to distance produced changes in apparent distance and lateral phoria. Positive lenses of $0, .5,2.0,3.5$, and 5.0 spherical diopters (SD) were used to manipulate the level of accommodative convergence in force during the period of maintained fixation. An inverse relationship was found between the stimulus to accommodation and the magnitude of the induced esophoria, the phoria being linearly related to an increase in apparent distance. The distance aftereffect obtained in the condition with the lowest net accommodative stimulus (i.e., $0 \mathrm{D}$ ) equaled that typically produced by base-out prism adaptation with full secondary cues to distance available. In a second experiment, subjects walked through a well-lit hallway while viewing through a pair of $5^{\Delta}$ base-out prisms. It was shown that increasing the stimulus to accommodation by adding negative lenses of $0,1.5,3.5$, and 5.5 SD reduced the adaptive change in apparent distance, as well as the change in phoria produced by the conventional base-out prism adaptation paradigm. It was concluded that a change in the resting tonus of the disparity vergence system underlies such adaptation, rather than recalibration of the oculomotor cues to distance. Monocular exposure data indicated that a small change in the tonus control for the accommodative system may be present as well.
\end{abstract}

Adaptation of perceived distance mediated by oculomotor cues was first investigated by Wallach and Frey (1972a). The method entails wearing spectacles with prism and lens combinations that effect either an increase or a decrease in the optical distance to targets. The result of relatively short viewing periods, of, say, 15 to $20 \mathrm{~min}$, is sufficient to alter perceived distance, and hence also the apparent size and depth of test targets (Wallach, Frey, \& Bode, 1972). Since apparent distance is increased after exposure to a shortened optical distance and decreased after exposure to a lengthened optical distance, the effect may be regarded as adaptive, in the sense that the perceptual result serves to counteract the immediate effects of the optically imposed transformation.

Two theoretical interpretations of the cause of the phenomenon have been proposed. The cue-discrepancy theory proposed by Wallach (1976) requires the presence of two or more cues (stimulus conditions) that normally lead to the same perceptual end-state. When the end-state associated with a subset of the total available cues is made to vary, as with the use of prisms, a cue conflict is said to exist, and this state represents the basis for perceptual adaptation. The latter occurs through a process of perceptual learning in which one cue becomes "reeducated" so as to elicit a percept more closely allied to that of the remaining

This research was supported in part by NIH Grants EY02264 and EY03421 to S.M.E. The authors' mailing address is: Department of Psychology, University of Wisconsin, Madison, Wiscon$\sin 53706$. cues than previously. When prism and lens combinations that decrease optical distance are worn, the oculomotor distance cues provided by vergence and accommodation signal a distance magnitude that is less than that of other cues not influenced by the optical system. Such remaining cues as motion parallax, texture gradients, linear perspective, familiar size, etc., continue to cue distance as usual and presumably serve to alter the cue function of the oculomotor system. The net result is thought to be "a recalibration in the relation between oculomotor adjustment and registered distance" (Wallach, Frey, \& Bode, 1972, p. 116). In the specific case of diminished optical distance, according to the theory, a given magnitude of convergence and accommodation will come to represent distance greater than that signaled prior to the cue-discrepancy experience.

The second interpretation is based upon physiologic properties of the oculomotor system itself and, in contrast to the recalibration theory, requires no assumptions about learning or even that a re-pairing between oculomotor innervation and perception is necessary. At the heart of this approach is a hysteresislike phenomenon associated with the control of tonus of the extraocular muscles. In general, when eye position either is maintained in a fixed posture (Ebenholtz \& Wolfson, 1975; Park, 1969; Schor, 1979a) or is exercised in asymmetrical postures (Shebilske, 1977), subsequent measures of the resting level such as the phoria or "straight-ahead" eye position reveal a consistent eye-position bias. Because the bias, which persists when the inducing conditions are no longer 
present, is always in the direction in which the eyes were last held, and because it can be brought to zero only by reversing the posture or asymmetry of the inducing ocular movements, ${ }^{1}$ it has been termed a hysteretic phenomenon, by analogy with instances of physical hysteresis (Ebenholtz, 1981; Shebilske, 1977).

The perceptual implications of ocular-motor hysteresis for several forms of prism adaptation such as eye-specific adaptation, displacement, and distance adaptation have been suggested previously (Ebenholtz, 1970, 1974; Paap \& Ebenholtz, 1976, 1977; Welch, 1978). In the case of distance adaptation, the central fact simply is that adaptation induces a shift in resting level of convergence as measured, for example, by the lateral phoria (Ebenholtz, 1981; Schor, 1979b). It follows that, in order to verge on a particular target, the requisite level of innervation of the extraocular muscles will be a function of the target distance as well as the level and direction of induced phoria. From these considerations, a distance illusion or adaptation aftereffect can be deduced with the aid of the premise that the innervation used to overcome (or complement) the induced phoria is "read" by the system as a change in perceived distance.

No change is posited in the calibration function that relates a given convergence posture and the associated perceived distance. All that changes after adaptation is the innervation level (or muscle tonus) needed to maintain binocular fixation on a target, due to the necessity to compensate for the induced phoria.

The mechanism underlying the induced phoria, its function in the control of vergence movements, and the factors that condition the phoria magnitude can best be understood in the context of a control system model fashioned after the work of Krishnan and Stark (1977) and Schor (1979a, 1980). In Figure 1 are represented two components of the triple synkinesis that normally exists between vergence, accommodation, and pupillary response, with the latter omitted.

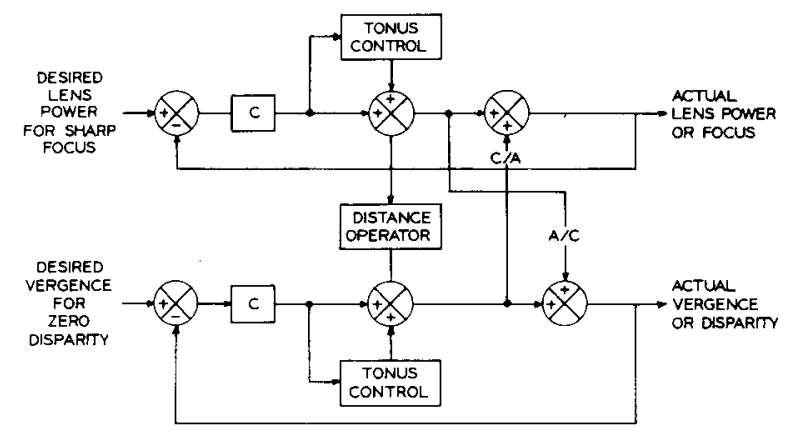

Figure 1. Elements of a control system model showing relationshlps between vergence and accommodation systems.
Both vergence and accommodation are governed by a rapid error-correcting feedback mechanism whose controllers are labeled " $C$ "' in the diagram. Each system is, in addition, served by a feed-forward signal from the opposite system, producing accommodative convergence $(A / C)$ and convergent accommodation $(\mathrm{C} / \mathrm{A})$, respectively. The lateral phoria, when measured after opening the disparity vergence loop by covering one eye, reflects both the adequacy of the $A / C$ response and the level of open-loop tonic innervation in the convergence system. The two sources labeled "tonus control" are thought to control the steady state innervation to the ciliary muscles in the accommodation loop and to the extraocular muscles in the vergence system. Although in the former case the tonus control mechanism is largely hypothetical, in the latter instance there is good empirical support for a slow vergence component with a long time constant (Schor, 1980). The tonus control mechanism may be the locus of control of the physiologic position of rest, that is, the dark vergence (Owens \& Leibowitz, 1980), and is proposed as the source of distance adaptation and aftereffects (Ebenholtz, 1981).

The model represented in Figure 1 presumes that distance perception is derived primarily from the steady-state levels of convergence and accommodation, and hence the role of the "distance operator" is to extract this information and to assign proper weights to the two sources. When both accommodation and convergence are in their respective resting positions, it is assumed that they correspond to a set of reference distances, and when departures from these resting states occur, there is a corresponding increment or decrement in distance perception. This is, therefore, a "set-point" theory similar to proposals made by Foley (1980) and von Hofsten (1976) in which a reference egocentric distance is posited. In the present model, the reference, or set-point, is identified with the mechanisms responsible for the steady-state tonus control. Perceptual adaptation is inferred as a direct outgrowth of changes in the reference level or system set point.

Previous research (Ebenholtz, 1981) has shown that variables that influence the level of distance adaptation, such as monocular vs. binocular exposure and induction vs. adaptation paradigms, also produce proportional shifts in the lateral phoria, as the model requires. Also consistent with the model is the finding of proportional shifts in distance adaptation and measures of the dark vergence (Owens \& Leibowitz, 1980).

In the adaptation paradigm, subjects wear prismlens combinations, usually while walking in a typical indoor environment rich in monocular cues, whereas in the induction paradigm, a luminous target is fixated in otherwise total darkness. In the latter case, although distance adaptation may readily be found, 
it is typically lower than that obtained under the adaptation paradigm. It may be thought, therefore, that veridical distance cues such as those present in the adaptation paradigm actually do serve to recalibrate the oculomotor cues (e.g., Wallach \& Halperin, 1977). The present experiments demonstrate that there are conditions under which the induction paradigm (Experiment 1) matches the adaptation paradigm (Experiment 2) in the level of adaptation and that, therefore, monocular distance cues are unnecessary to the process. Experiments also will show that a critical variable capable of modulating the level of distance adaptation and lateral phoria is the level of accommodative convergence in force during the exposure period. As Figure 1 shows, high levels of A/C may be expected to lower the activity of the disparity vergence controller $(C)$, with less of a consequent input to the tonus control mechanism, and hence less of a change in distance perception to be expected.

Schor (1979a) has already shown that these results apply to the lateral phoria for short-term fixation periods, up to $1 \mathrm{~min}$, but implications of the model for the interrelationships between long-term prism adaptation, induced phoria, and distance perception remain to be tested. In Experiment $1, \mathrm{~A} / \mathrm{C}$ was manipulated in the context of an induction paradigm by systematically decreasing the stimulus to accommodation while disparity vergence was held constant.

\section{EXPERIMENT 1}

\section{Method}

Design. Each of five groups of six subjects each was assigned to one of five conditions in which the subjects binocularly fixated a luminous target while viewing through positive lenses of $0, .5,2.0$, 3.5 , or 5.0 spherical diopters (SD). Since the target was at $20 \mathrm{~cm}$, these conditions provided, respectively, $-5,-4.5,-3.0,-1.5$, and 0 SD stimulus to accommodation. The subjects were assigned to conditions according to appearance at the laboratory and were alternated among conditions after every two subjects.

Apparatus and Procedure. The first activity of all subjects was a walk in the hallway for two cycles around the laboratory wing lasting about $5 \mathrm{~min}$. This served to mitigate any short-term vergence adaptation (e.g., Schor, 1979a) that might have been triggered by near work activities, such as reading in the period immediately preceding the experiment. This was followed by a measure of the interocular axis and the lateral phoria. The latter measure was taken with a Green's refractor and a target consisting of a red LED with cross hairs, at $33.3 \mathrm{~cm}$ from the front surface of the cornea. The optics of the measurement procedure are represented in Figure 2. In front of the left eye was a set of Risely prisms at $\mathbf{0}$ prism diopters (PD) and a clear Maddox rod that caused the LED to project as a vertical red line. The right eye viewed the target through a pair of prisms also at $O$ PD so as to equate the slight magnifying effect of the glass between the two eyes. After placing his or her chin in the chin cup behind the refractor, the subject was told to maintain fixation on the cross hairs over the red dot, to report whether the line was located to the right or left of the dot, and to report when it was centered in the dot. The left eye was uncovered for about a $.5-\mathrm{sec}$ interval and then occluded, during which time the experimenter adjusted the prisms so as to bring the line into the same apparent direction as the dot. Exophoria was indicated if the line was initially seen to the right of the dot, whereas

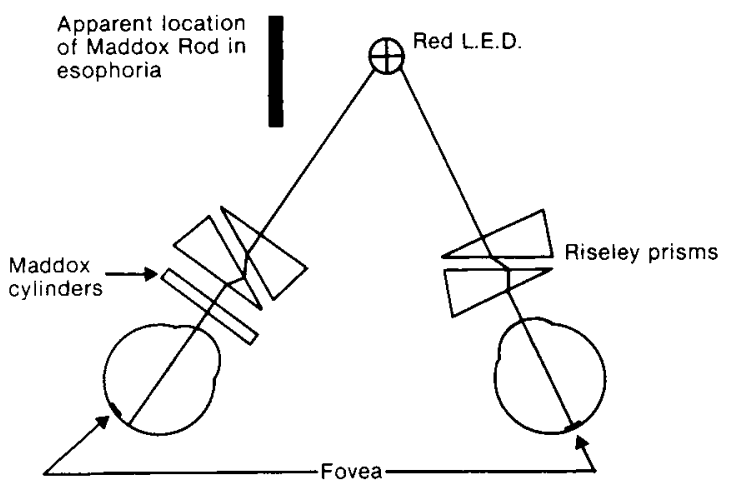

Figure 2. In esophoria, the nonfixating eye is overconverged. Phorla magnitude is equal to the prism displacement needed to place the image of the Maddox rod at the retinal location corresponding to the image of the target in the fixating eye.

esophoria caused the line to be located to the left of the dot. Phoria testing, measured to the nearest diopter, was accomplished quite rapidly, usually within about $1 \mathrm{~min}$.

Exophoria is the normal physiologic state at near target distance (Duke-Elder \& Wybar, 1973). Consequently, subjects exhibiting esophoria, or an exophoria in excess of $16 \mathrm{PD}$ on the pretests, did not participate further in the experiment.

Upon completion of the phoria measure, with the subject's eyes closed, the refractor head was displaced and a foreheadrest was substituted in preparation for a manual measure of apparent distance. To do this, the subject moved a slide with the index finger of the unseen preferred hand until it matched the position of a luminous target, shown in Figure 3. The target, viewed binocularly in darkness, was stationed at eye level, $33.3 \mathrm{~cm}$ from the anterior surface of the cornea. Two measures were taken, one each from starting positions at $10 \mathrm{~cm}$ on either side of the true position. Prior to the main test trials, four practice trials (two settings each) were given with the target at $30.0,38.5,35.9$, and $31.0 \mathrm{~cm}$. The mean of the two settings at each target position was used as the subject's score. If mean errors exceeded $20 \mathrm{~cm}$ or the subject failed to discriminate among any of the four practice targets, he or she was eliminated from the study.

A 15-min exposure period followed the manual distance measure, in which a luminous target at $20 \mathrm{~cm}$, shown in Figure 4, was

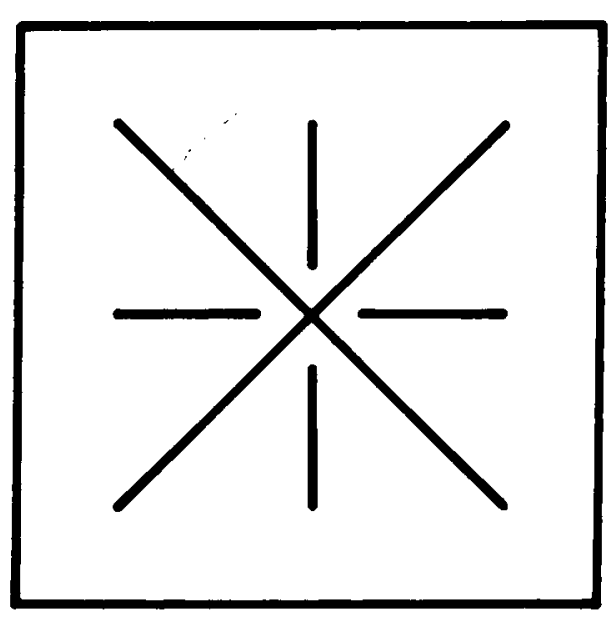

Figure 3. Target used on manual distance tests. Outer square is $12 \times 12 \mathrm{~mm}$; diagonal lines are $10 \mathrm{~mm}$ long. 


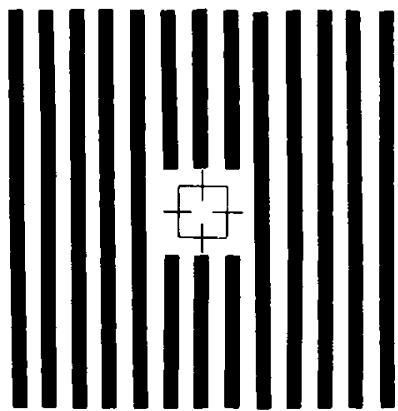

Figure 4. Fixation target used during the induction procedure. Black areas are luminous. The outer square was $4 \times 4 \mathrm{~cm}$; the inner square was $.5 \times .5 \mathrm{~cm}$. Bars were $1.6 \mathrm{~mm}$ wide.

binocularly fixated through a pair of positive spherical lenses with power as described above. The bars surrounding the central square pattern constituted a square-wave frequency of 1.1 cycles deg $^{-1}$ and were useful in maintaining fusion while the lenses relaxed accommodation.

Immediately after the exposure period, the lenses were removed and there followed two settings on the manual distance test, a phoria measure, and a test for visual acuity on the Bausch and Lomb Orthorater for near targets (i.e., $33.3 \mathrm{~cm}$ ). In units corresponding to a 20-ft test distance, scores over both eyes for all but two subjects ranged from $20 / 17$ to $20 / 22$. One subject scored at $20 / 20$ and $20 / 25$ and another scored at $20 / 25$ and $20 / 22$ over the left and right eye, respectively.

Subjects. The participants were students who had volunteered and had no known history of oculomotor or other visual disorders and no prescribed correction. Eight subjects were replaced in the study for excessive or inadequate performance on the preexposure tests. Reasons included evidence of suppression in one eye (one subject), esophoria of 8 PD (one subject), exophoria of -22 PD (one subject), pointing on the manual distance test by $20 \mathrm{~cm}$ or more beyond the target (two subjects), and inability to maintain fusion during exposure (three subjects).

\section{Results and Discussion}

Preexposure. The five groups did not differ on the preexposure distance settings $[F(4,25)=.93, p>.05]$. Over all 30 subjects, the mean overreaching error was $5.5 \mathrm{~cm}$ for the target at $33.3 \mathrm{~cm}$, an error commonly found in this type of task (Ebenholtz, 1981; Foley, 1975). There were likewise no differences among the groups on the magnitude of the preexposure lateral phoria measurements $[\mathrm{F}(4,25)$ $=.40, \mathrm{p}>.05]$. On average, subjects exhibited
-7.6 PD (exophoria), a value within the orthophoric range at near test distances (Duke-Elder \& Wybar, 1973).

Postexposure. The change in phoria and in distance settings as a result of the 15 -min fixation period are presented in Table 1. The mean post-pre shifts in phoria were all in the esophoric direction and differed significantly across conditions $[F(4,25)=4.35$, $\mathrm{p}<.01]$, as did the differences in pointing responses $[F(4,25)=5.57, p<.01]$. Note that in the condition requiring maximum accommodative relaxation ( 0 net stimulus to accommodation) with no prisms worn, the distance aftereffect is equivalent to that obtained in a $15-20$-min prism adaptation paradigm, usually with full secondary cues available (Ebenholtz, 1981; Wallach, Frey, \& Bode, 1972).

The function relating the changes in phoria to the net stimulus to accommodation is shown in Figure 5, where a linear function has been fit to the data. The esophoria induced as a result of target fixation decreases linearly with increases in the stimulus to accommodation. Even with the aberrant point ${ }^{2}$ at $-5.0 \mathrm{SD}$, the linear fit is excellent, yielding an $r$ of .96. Thus, it is clear, as Figure 1 illustrates, that the more relaxed the accommodation, the greater will be the induced change in esophoria as required by the proportional decrease in accommodative convergence. The rate of change in induced esophoria per unit shift in accommodation exposure is expressed as the slope of the fitted function. This corresponds to a group mean accommodative convergence per unit of accommodation ratio, that is, an aftereffect $\mathrm{AC} / \mathrm{A}$ ratio of 1.5 $\mathrm{PD} \mathrm{SD}^{-1}$. Unlike the conventional $\mathrm{AC} / \mathrm{A}$ ratio (Alpern, 1962), in which increases in the stimulus to accommodation cause increases in accommodative convergence, the aftereffect $\mathrm{AC} / \mathrm{A}$ ratio reflects the decrease in disparity-vergence aftereffect associated with the increased accommodative convergence as the stimulus to accommodation increases. This reciprocal relation between the two $\mathrm{AC} / \mathrm{A}$ ratios is precisely as required by the model represented in Figure 1.

In order to evaluate the relation between changes in esophoria and distance settings, the two measures

Table 1

Mean Changes in Phoria (PD) and in Distance Settings (in Centimeters) According to the Net Stimulus to Accommodation (SD) During the Exposure Period

\begin{tabular}{|c|c|c|c|c|c|c|c|c|c|c|}
\hline & \multicolumn{10}{|c|}{ Net Accommodative Stimulus (SD) } \\
\hline & \multicolumn{2}{|c|}{0} & \multicolumn{2}{|c|}{-1.5} & \multicolumn{2}{|c|}{-3.0} & \multicolumn{2}{|c|}{-4.5} & \multicolumn{2}{|c|}{-5.0} \\
\hline & $\mathbf{M}$ & $\sigma_{\mathrm{M}}$ & $\mathrm{M}$ & $\sigma_{\mathrm{M}}$ & $\mathbf{M}$ & $\sigma_{M}$ & M & $\sigma_{\mathrm{M}}$ & M & $\sigma_{\mathbf{M}}$ \\
\hline Phoria & $10.33^{*}$ & 2.16 & $7.58^{*}$ & 1.91 & $4.50^{*}$ & 1.29 & $2.17^{*}$ & .65 & $3.67 *$ & 1.41 \\
\hline Distance & $11.33^{*}$ & 1.59 & $5.28^{*}$ & .77 & $6.63^{*}$ & 2.05 & $3.32 *$ & .96 & $3.34 *$ & 1.24 \\
\hline
\end{tabular}

Note-Positive scores indicate a shift toward esophoria and an increase in pointing distance. ${ }^{*}$ Significant at $p=.05$ or better. 


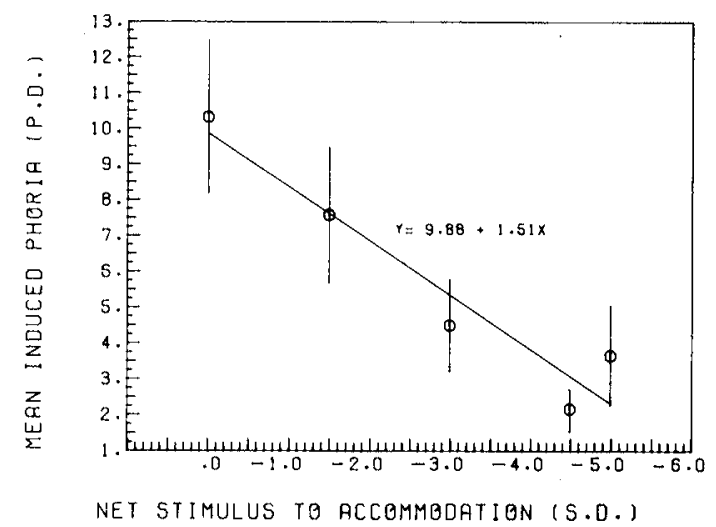

Figure 5. Mean induced esophoria as a function of the net stimulus to accommodation. Vertical segments represent \pm 1 standard error. Continuous line is the best-fit linear function.

were transformed into a common metric, namely, convergence distance expressed in degrees. Convergence distance based upon the induced esophoria was determined by subtracting the postexposure change in phoria from the actual convergence angle of each subject when fixating the test target at $33.33 \mathrm{~cm}$. For example, a subject with an interocular axis of $6.20 \mathrm{~cm}$ who binocularly fixates a target at $33.33 \mathrm{~cm}$ does so with a convergence angle of $10.64 \mathrm{~cm}$. If this subject showed a change of $+10 \mathrm{PD}$ (eso), then $5.73 \mathrm{deg}$ was subtracted from the subject's actual convergence angle, yielding $4.91 \mathrm{deg}$. The result was a hypothetical convergence angle generated "as if" the subject were fixating a point beyond the actual target by an amount precisely enough to compensate for the induced esophoria. Convergence distance based upon manual distance settings was calculated by adding the postexposure shift in distance settings to the actual fixation distance of $33.33 \mathrm{~cm}$ and then computing each individual's convergence angle based upon this "effective" distance. However, since the relation between pointing distance and actual target distance, although linear, was shown to have a slope greater than 1 (Ebenholtz, 1981), it was necessary to calibrate the pointing increments against true distances before calculating the final convergence distance. In order to accomplish this, a weighting factor was determined by taking the difference in preexposure manual settings to the farthest practice target at $38.5 \mathrm{~cm}$ and the test target at $33.3 \mathrm{~cm}$, and then dividing by the true difference of $5.2 \mathrm{~cm}$. This yielded an average ratio over all subjects of $1.35: 1 .^{3}$ Each pointing increment was therefore reduced by a factor equal to the reciprocal of this number, that is, by .741 . According to this procedure, a subject whose pointing distance increased by, for example, $10 \mathrm{~cm}$, as a result of the fixation period behaves as though he were pointing at a target that was displaced by $7.41 \mathrm{~cm}$ from its preexposure position. For this hypothetical subject, the "effective" convergence distance equaled $40.74 \mathrm{~cm}$ (i.e., $33.33+7.41$ ) which, for an interocular axis of $6.20 \mathrm{~cm}$, corresponds to a convergence angle of $8.70 \mathrm{deg}$.

Figure 6 represents the relation between two independent estimates of convergence distance, viz., that based upon the manual response as ordinate against that inferred from the change in phoria on the $a b-$ scissa. The least square procedure applied to the mean values for each of the five groups yielded an excellent linear fit with an $r$ of .92 accounting for $85 \%$ of the variance. The adequacy of the fit is further demonstrated by the small standard error of estimate $\left(\sigma_{\text {est }}\right)$ of $.21 \mathrm{deg}$ and also by the fact that if a value of $x$ corresponding to the absence of any induced phoria, that is, $33.33 \mathrm{~cm}$, is substituted in the equation, the predicted value of the convergence distance based on pointing is $34.41 \mathrm{~cm}$. In terms of convergence angle, this value is only $.33 \mathrm{deg}$ from the correct angle of $10.41 \mathrm{deg}^{4}$ and within the margin of error only $1.57 \sigma_{\text {est }}$ units. When the equation is solved for $\mathrm{x}=0 \mathrm{deg}$ corresponding to convergence at optical infinity, a predicted convergence distance of $7.37 \mathrm{deg}$ or $47.12 \mathrm{~cm}$ is found. This result, and not a value closer to optical infinity, occurs because the slope of the regression line is less than one, which implies that only a portion of the induced phoria is expressed in the change in pointing distance. ${ }^{3}$ The obtained value suggests an upper limit to the adaptation of convergence distance after target fixation and with the use of plus-lenses under the conditions of the present study.

The results of both analyses show an increasing induced esophoria that varies inversely with the stimulus to accommodation and an increase in apparent distance linearly related to the phoria magnitude. Since the distance aftereffect obtained with the lowest net stimulus to accommodation was equivalent in magnitude to that obtained under conven-

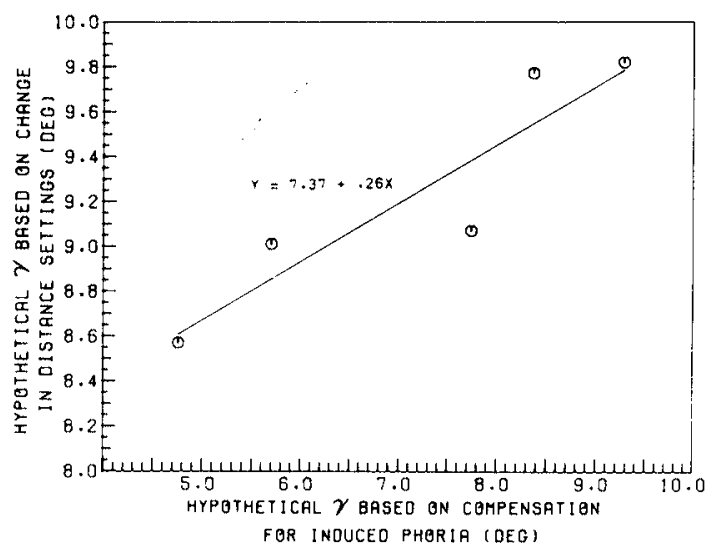

Figure 6. Relationship between predicted convergence angle ( $\gamma$ ) based on calibrated distance settings (ordinate) and that based on compensation for the induced phorla (abscissa). Continuous line is the best-fit linear function. 
ventional adaptation procedures (e.g., in a lighted hallway with base-out prisms), even though it occurred in the absence of secondary distance cues, the latter cannot be necessary for distance adaptation to occur.

In Experiment 2 in an adaptation paradigm, the stimulus to accommodation was systematically increased with minus-lens additions and was accompanied by a constant disparity-vergence increment produced by base-out prisms. In this paradigm, adaptation occurred in the context full of monocular (secondary) cues to distance and hence exposure conditions correspond closely to those used in previous distance adaptation studies (e.g., Ebenholtz, 1974, 1981; Wallach \& Frey, 1972a). In this case, as Figure 1 suggests, increasing accommodative convergence should once again lead to a decrease in the input to the vergence tonus control center, and hence a drop in vergence adaptation should occur.

\section{EXPERIMENT 2}

\section{Method}

Design. In a within-subjects design, each of eight subjects was exposed, binocularly, to four levels of increasing accommodative power while wearing a pair of $5-\Delta$ base-our prisms. Lenses were $0,-1.5,-3.5$, and $-5.5 \mathrm{SD}$ and were worn by the subjects as they walked through a hallway in 15 -min sessions separated by at least $48 \mathrm{~h}$. The order of exposure was determined by a completely balanced four-item Latin square, two subjects assigned to each sequence. Seven of the eight subjects returned for two additional 15-min monocular sessions in which no prisms but only a 0 - or -5.0-SD lens was worn over the preferred eye. The order in which the lenses were worn was alternated over successive subjects. A minimum of $48 \mathrm{~h}$ intervened between sessions.

Apparatus and Procedure. First, the interocular separation was measured to the nearest half millimeter, using a Titmus P-D scope. This was followed by a test of eye dominance based upon the pointing test (Coren \& Kaplan, 1973) with a target at about $2.4 \mathrm{~m}$. The phoria was then measured as described in Experiment 1, with the preferred eye fixating the cross hairs. This was followed in order by binocular and monocular manual distance tests and the 15-min exposure period. Immediately after the exposure session, the phoria again was measured with added lenses and prisms removed, followed by binocular and monocular distance tests. At the end of the very first exposure period, far visual acuity was measured in the Bausch and Lomb Orthorater, and no less than $24 \mathrm{~h}$ after the last session the AC/A ratio was taken. For this purpose, the subject's phoria was again measured with the target at $33.33 \mathrm{~cm}$, followed by a second measure taken with the addition of a +2.0-D lens over the preferred eye. The AC/A ratio was calculated by dividing the change in phoria by the difference in lens power (Ogle \& Martens, 1957).

For the two monocular sessions, the test sequences were the same as that used in the binocular sessions, except for the postexposure sequence in which the monocular manual distance test preceded the binocular distance test.

Subjects. The subjects were student volunteers who reported no known oculomotor disorder and had no prescribed correction. One subject was replaced, because of a low AC/A ratio of .5 PD $\mathrm{SD}^{-1}$. The same subject was unable to maintain a clear focus during the exposure session with the -3.5-SD lenses.

\section{Results and Discussion}

Preexposure (binocular sessions). Phoria scores did not vary significantly over the four test sessions
$[F(3,21)=.975, p>.05]$ or when grouped according to the experimental conditions that they preceded $[F(3,21)=.975, \mathrm{p}>.05]$. There was, thus, no evidence of either practice effects or of a bias in phoria associated with any given condition. Over all four sessions and subjects, the phoria averaged -5.06 PD (exophoria).

Preexposure distance settings likewise gave no evidence of practice effects over the four sessions $[F(3,21)=.121, p>.05]$. Nor did the subjects vary when grouped according to the lens power they subsequently received $[F(3,21)=1.885, p>.05]$. Over subjects and conditions, distance settings to the $33.33 \mathrm{~cm}$ test target averaged $39.12 \mathrm{~cm}$.

Far acuity scores ranged from $20 / 17$ to $20 / 22$, and the $\mathrm{AC} / \mathrm{A}$ ratio ranged from 1.25 to 3.00 .

Postexposure (binocular sessions). The change in phoria and in distance settings as an aftereffect of each of the four 15-min exposure sessions is represented in Table 2. Consistent with the use of base-out prisms, phoria change always was in the esophoric direction and differed significantly, as predicted, across exposure conditions $[\mathrm{F}(3,21)=10.23, \mathrm{p}<$ .01]. The function relating lens power to the shift in phoria is represented in Figure 7, in which a linear function has been fit to the data. An excellent fit resulted with $r=.970$. Thus, although the actual stimulus to accommodation varied as a function of the particular target observed in the hallway, the esophoria diminished regularly with the increment in negative lens power. The slope of the function, 1.3 PD SD ${ }^{-1}$, is remarkably close to that found with the induction procedure of Experiment 1 shown in Figure 4 (i.e., 1.5 PD SD ${ }^{-1}$ ) and clearly indicates that common processes underlie both the induction and adaptation paradigm.

Changes in pointing distance, measured under binocular test conditions, are represented in Table 2. Apparent distance varied significantly with lens power present in the exposure sessions $[F(3,21)=$ 5.57], decreasing with increasing negative stimulus to accommodation. The relationship between changes in pointing distance and induced phoria was analyzed, as described previously, ${ }^{6}$ and is represented in Figure 8. The linear fit yielded an $r$ value of .854 . When the convergence angle corresponding to the

Table 2

Mean Change in Phoria (PD) and Binocular Distance Settings (Centimeters) by Lens Power (SD) During Each Binocular Exposure Session

\begin{tabular}{|c|c|c|c|c|c|c|c|c|}
\hline & \multicolumn{8}{|c|}{ Lens Power (SD) } \\
\hline & \multicolumn{2}{|l|}{0} & \multicolumn{2}{|c|}{-1.5} & \multicolumn{2}{|c|}{-3.5} & \multicolumn{2}{|c|}{-5.5} \\
\hline & $\mathbf{M}$ & $\sigma_{\mathrm{M}}$ & $\mathbf{M}$ & $\sigma_{\mathrm{M}}$ & $\mathbf{M}$ & $\sigma_{\mathrm{M}}$ & $\mathbf{M}$ & $\sigma_{\mathrm{M}}$ \\
\hline Phoria* & $10.31 * *$ & .95 & $8.25^{* *}$ & 1.00 & $4.63 * *$ & 1.07 & $3.63^{* *}$ & $=1.07$ \\
\hline Distance & $8.94 * *$ & 1.17 & $9.63 * *$ & 2.06 & $7.02 * *$ & 1.73 & $4.30^{* *}$ & 1.23 \\
\hline
\end{tabular}

*Positive scores indicate a shift toward esophoria and an increase in pointing distance. **Significant at $p=.05$ or better. 


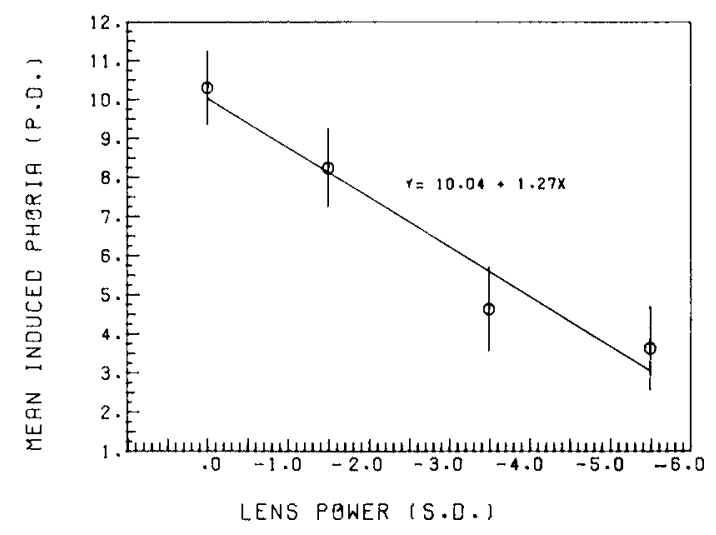

Figure 7. Mean induced esophoria as a function of lens power during adaptation. Vertical bars represent \pm standard error. Continuous line is the best-fit linear function.

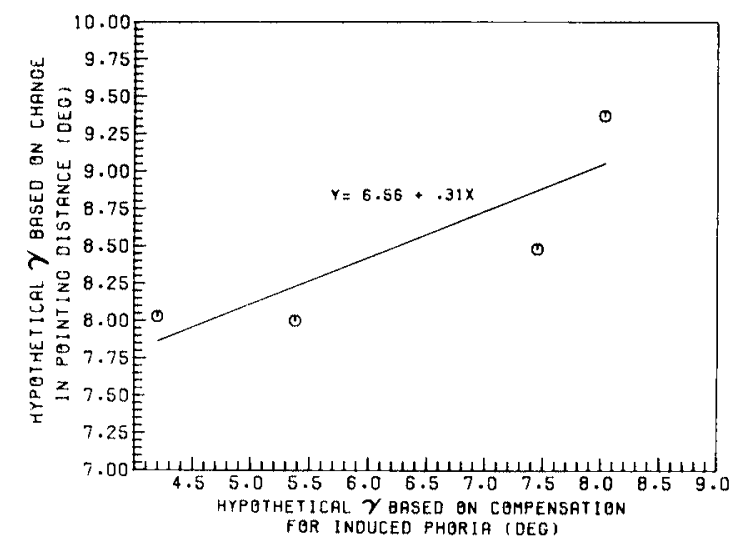

Figure 8. Relationship between predicted convergence angle $(\gamma)$ based on calibrated distance settings (ordinate) and that based on compensation for the induced phoria (abscissa). Continuous line is the best-fit linear function.

absence of an induced phoria, that is, $10.10 \mathrm{deg}$ $(33.33 \mathrm{~cm})$, is substituted in the linear equation, a convergence angle of $9.69 \mathrm{deg}(34.74 \mathrm{~cm})$ is obtained. This is within $1.24 \sigma_{\text {est }}$ units of the proper angle and is indicative of a reasonably good fit. Substituting a value of zero, which would come about only if the induced phoria were equal to the convergence angle at $33.33 \mathrm{~cm}$, yielded a predicted convergence of $6.56 \mathrm{deg}$, or $51.39 \mathrm{~cm}$. This is consistent with the data of the induction procedure, since a shift of $18.06 \mathrm{~cm}$, and not an infinite increment, is predicted. Thus, both paradigms seem to require a limited effect ${ }^{s}$ in accordance with the actual data, indicating asymptotic performance (Ebenholtz, 1981), and in both cases the limit is set by the magnitude of the induced phoria.

Monocular testing. The subjects did not differ on the preexposure monocular distance tests when grouped according to subsequent binocular exposure conditions $[F(3,21)=.897, p>.05]$. After exposure, small differences in pointing responses occurred on the monocular test, but these did not differ significantly as a function of exposure conditions $[F(3,21)=1.38$, $\mathrm{p}>$.05]. Averaged over sessions and subjects, the mean monocular distance increment after binocular exposure was significantly different from zero at $2.84 \mathrm{~cm}[\mathrm{t}(7)=3.64, \mathrm{p}<.01]$. It is clear that the disparity vergence system must be implicated at the time of the test for large changes in pointing distance to occur, but there is nevertheless a slight monocular component. This was explored in the final exposure sessions, in which monocular viewing through 0 and $-5.5 \mathrm{SD}$ was compared.

Monocular exposure. The subjects did not differ on either the preexposure phoria test $[\mathrm{t}(6)=.506, \mathrm{p}>$ $.05]$ or the predistance tests [t $(6)=.369$ (monocular test) and $\mathrm{t}(6)=.706$ (binocular test) associated with the two subsequent exposure conditions]. After exposure, for neither condition was the change in phoria significantly different from zero, although the difference in phoria shift between the 0 and -5.5 SD lenses was significant $[t(6)=2.30, p<.05]$. These data and the pointing difference scores are represented in Table 3. Shifts in pointing distance under binocular and monocular test conditions both were significant, but only after the -5.5-SD condition, and only in the binocular case was the shift significantly different from that in the 0-SD condition. It is clear that monocular exposure with a $-5.5-\mathrm{D}$ lens is sufficient to produce a significant shift in pointing distance. Although the mean scores suggest that binocular testing yields higher distance shifts than monocular tests, statistical analysis did not bear this out.

After monocular exposure, a small, but significant, distance aftereffect has been demonstrated with the -5.5-SD lens. Yet, under binocular exposure, this condition was expected to yield the lowest aftereffect. Reasoning from the monocular exposure data, we propose that, as accommodative convergence increases, the binocular aftereffects should approach a lower limit, due primarily to the accommodative component. This is supported by the fact that, when distance changes were compared under

Table 3

Mean Change in Phoria (PD) and Distance Settings (Centimeters) After Monocular Exposure Under Monocular and Binocular Testing

\begin{tabular}{|c|c|c|c|c|c|c|}
\hline \multirow{3}{*}{$\begin{array}{c}\text { Lens } \\
\text { Power } \\
\text { (SD) }\end{array}$} & & & \multicolumn{4}{|c|}{ Distance Tests } \\
\hline & \multicolumn{2}{|c|}{ Phoria* } & \multicolumn{2}{|c|}{ Monocular } & \multicolumn{2}{|c|}{ Binocular } \\
\hline & M & $\sigma_{M}$ & M & $\sigma_{M}$ & M & $\sigma_{\mathrm{M}}$ \\
\hline $\begin{array}{c}0 \\
-5.5\end{array}$ & $\begin{array}{r}-1.57 \\
1.71\end{array}$ & $\begin{array}{l}1.09 \\
1.89\end{array}$ & $\begin{array}{l}.89 \\
2.77 * *\end{array}$ & $\begin{array}{l}1.43 \\
1.17\end{array}$ & $\begin{array}{c}.66 \\
4.44^{* *}\end{array}$ & $\begin{array}{r}1.59 \\
.91\end{array}$ \\
\hline
\end{tabular}

*Positive scores indicate a shift toward esophoria and an increase in pointing distance. $\quad * *$ Significant at $p=.05$ or better. 
binocular testing in the $-5.5-\mathrm{SD}$ conditions, binocular and monocular exposure showed equivalent effects at 4.30 and $4.44 \mathrm{~cm}$, respectively $[\mathrm{t}(6)=.32$, $\mathrm{p}>.05]$.

\section{GENERAL DISCUSSION}

Experiment 1 has shown that the induction procedure is capable of yielding high levels of distance adaptation, in the absence of secondary cues to distance. Hence, the latter cannot be necessary for distance adaptation. Experiment 2 has shown that even in the presence of monocular distance cues in the conventional adaptation paradigm, distance adaptation can easily be modulated by manipulating accommodative convergence. Hence, secondary distance cues cannot be sufficient to produce distance adaptation, except possibly for the very lowest levels of adaptation obtained. But even in this case, since monocular exposure to negative lenses provides a basis for accommodation as opposed to vergence aftereffects, there remains very little, if any, adaptation for which distance cues must account.

There is, however, a role for distance cues in the adaptation process, although it is not to recalibrate the oculomotor cues. The present experiments have shown that both adaptation and induction paradigms cause an induced lateral esophoria that serves to predict the magnitude of distance adaptation. In both cases, the phoria and the level of distance adaptation was increased by decreasing the stimulus to accommodation. This, in turn, lowered the level of accommodative convergence and thereby enhanced the output of the tonus control mechanism of the disparityvergence loop. Thus, the role of distance cues in a well-structured environment may be thought of as serving the same function as positive lenses, namely, to relax the level of accommodative convergence. Such a role for distance cues is generally accepted and referred to as a "proximal" component of accommodation control responsive to psychological factors mediating distance perception (Ittelson \& Ames, 1950). This analysis provides an account of the results of a recent distance adaptation study by Owens and Leibowitz (1980). They found the lowest levels of shift in both dark vergence ${ }^{7}$ and distance adaptation among a group of subjects who read text while others either walked and engaged in various sensorimotor activities or were passively translated through a hallway. Since the readers of necessity would have utilized the highest levels of accommodation and have little opportunity to take advantage of the hallway cues to relax accommodation and accommodative convergence, it is to be expected that the levels of adaptation and dark vergence would be correspondingly low. The remaining two groups may be expected to perform equivalently, as in fact they did.
It follows that the stimulus to accommodation, not the level of sensorimotor activity governs the levels of adaptation in these contexts.

The important role of accommodation and accommodative convergence also represents one of the reasons for the failure of Wallach and Halperin (1977) to obtain distance aftereffects in an induction paradigm. One group moved the head laterally to view binocularly and in succession $\$ 1$ bills placed at optical distances of $-3.5,-2.5$, and -1.79 SD. A second group viewed three luminous disks with a thin vertical wire stretched across them in place of the dollar bills, but with head constrained, thereby requiring a relatively greater magnitude of lateral eye movements. Both groups wore spectacles containing base-out prisms (5 PD) and -1.5-SD lenses. No phoria measurements were taken, but distance adaptation was assessed using a size-estimation task. Only the former group evidenced adaptation, and hence Wallach and Halperin concluded that the effect was attributable to the presence of veridical distance cues, in the form of the dollar bills, and other monocular (or secondary) cues as well. The present results and those of other studies (Schor, 1979b) demonstrate a likely artifact in these studies in the failure to take account of the reciprocal relation between accommodative convergence and the disparity-vergence innervation. It is likely that the dollar bills' offering a relatively low-contrast stimulus actually relaxed accommodation (Charman \& Tucker, 1977), while the thin wire on a luminous background no doubt provided a good accommodative stimulus. Low adaptation under the latter condition ${ }^{8}$ and high adaptation in the former case are therefore to be expected, and the presence of veridical cues probably is irrelevant, as Experiment 2 of the present study shows.

The role of accommodation and accommodative convergence may again have been neglected in yet another study in which changes in distance perception were brought about by viewing a "luminous figure that objectively expanded as it moved toward $S$ and contracted as it moved away" (Wallach \& Frey, 1972b, p. 121). Wallach and Frey attributed the change to a "modification in the relation between oculomotor adjustment and registered distance" (p. 165), the latter presumably influenced by the inordinate rate of change in image size, relative to the actual distance traversed. Unfortunately, however, no control procedure was investigated in which size changes were produced without changes in distance or in which distance changes occurred but without experimentally manipulated size changes. Further to the point are the observations by Ittelson and Ames (1950) that continuous size changes produce changes in apparent distance and either or both of these factors produce corresponding changes in accommodation. Relaxation of accommodation with increasing 
apparent distance would, as discussed above, serve as the sufficient condition for adaptation in the disparityvergence system and account for the obtained changes in distance perception but without the necessity of reference to the notion of recalibration of conflicting distance cues.

The present experiments and previous research (Ebenholtz, 1981) have shown a close association between distance perception and the change in lateral phoria, while other studies have demonstrated a similar relation between perceived distance and the resting level of convergence (Owens \& Leibowitz, 1980). The present studies have, in addition, demonstrated a reciprocal relation between the magnitude of accommodative convergence and change in distance perception under binocular test conditions. These results strongly support the view that accommodative convergence affects distance perception only insofar as it interacts with the disparity-vergence system. This conclusion also is consistent with the results of Schor (1979b) on the reciprocal relation between accommodative convergence and the change in phoria.

If it is true that the presence of a lateral phoria as such is irrelevant to apparent distance under monocular viewing conditions, then the perception of distance under monocular observation must be mediated by the tonus control mechanism regulating ciliary muscle tension. The obtained effects of monocular exposure on distance perception make it likely that there is an independent accommodative input to distance perception, as suggested in Figure 1.

\section{REFERENCES}

AlPE RN, M. Movements of the eyes. In H. Davson (Ed.), The eye (Vol. 3). New York: Academic Press, 1962.

Charman, W. N., \& Tucken, J. Dependence of accommodation response on the spatial frequency spectrum of the observed object. Vision Research, 1977, 17, 129-139.

Coren, S., \& Kaplan, C. P. Patterns of ocular dominance. American Journal of Optometry and Archives of American Academy of Optometry, 1973, 50, 283-292.

DUKe-Elde R, S., \& WYBAR, K. Ocular motility and strabismus. In S. Duke-Elder (Ed.), System of ophthalmology (Vol. 6). St. Louis: C. V. Mosby, 1973.

Ebenholtz, S. M. On the relation between interocular transfer of adaptation and Hering's law of equal innervation. Psychological Review, 1970, 77, 343-347.

Ebenholtz, S. M. The possible role of eye-muscle potentiation in several forms of prism adaptation. Perception, 1974, 3, 477-485.

Ebenholtz, S. M. Hysteresis effects in the vergence control system: Perceptual implications. In D. F. Fisher, R. A. Monty, \& J. W. Senders (Eds.), Eye movements: Visual perception and cognition. Hillsdale, N.J: Erlbaum, 1981.

Ebenholtz, S. M., \& Wolfson, D. M. Perceptual aftereffects of sustained convergence. Perception \& Psychophysics, 1975, $17,485-491$.

Foley, J. M. Error in visually directed manual pointing. Perception \& Psychophysics, 1975, 17, 69-74.
Foley, J. M. Binocular distance perception. Psychological Review, $1980,87,411-434$.

Ittelson, W. H., \& Ames, A. Accommodation, convergence, and their relation to apparent distance. Journal of Psychology, 1950, 30, 43-62.

Krishnan, V. V., \& Stark, L. A heuristic model for the human vergence eye movement system. IEEE Transactions on Biomedical Engineering, 1977, BME-24, 44-49.

Ogle, K. N., \& Martens, T. G. On the accommodative convergence and the proximal convergence. A.M.A. Archives of Ophthalmology, 1957, 57, 702-715.

OWENs, D. A., \& Leibowitz, H. W. Accommodation, convergence, and distance perception in low illumination. American Journal of Optometry and Physiological Optics, 1980, 57, 540-550.

PaAp, K. R., \& Ebenholtz, S. M. Perceptual consequences of potentiation in the extraocular muscles: An alternative explanation for adaptation to wedge prisms. Journal of Experimental Psychology: Human Perception and Performance, 1976, 2, 457-468.

PaAp, K. R., \& Ebenholtz, S. M. Concomitant direction and distance aftereffects of sustained convergence: A muscle potentiation explanation for eye-specific adaptation. Perception \& Psychophysics, 1977, 21, 307-314.

Park, J. N. Displacement of apparent straight ahead as an aftereffect of deviation of the eyes from normal position. Perceptual and Motor Skills, 1969, 28, 591-597.

Schor, C. M. The influence of rapid prism adaptation upon fixation disparity. Vision Research, 1979, 19, 757-765. (a)

Schor, C. M. The relationship between fusional vergence eye movements and fixation disparity. Vision Research, 1979, 19, 1359-1367. (b)

Schor, C. M. Fixation disparity: A steady state error of disparityinduced vergence. American Journal of Optometry and Physiological Optics, 1980, 57, 618-631.

Shebilske, W. Directional scanning biases and shifts of apparent visual direction. Vision Research, 1977, 17, 495-497.

von Hofsten, C. The role of convergence in visual space perception. Vision Research, 1976, 16, 193-198.

Wallach, H. Hans Wallach on perception. New York: Quadrangle, 1976.

Wallach, H., \& Frey, K. J. Adaptation in distance perception based on oculomotor cues. Perception \& Psychophysics, 1972, 11, 77-83. (a)

Wallach, H., \& Frey, K. J. On counteradaptation. Perception \& Psychophysics, 1972, 11, 161-165. (b)

Wallach, H., Frey, K. J., \& Bode, K. A. The nature of adaptation in distance perception based on oculomotor cues. Perception \& Psychophysics, 1972, 11, 110-116.

WAllach, H., \& HALPE RIN, P. Eye muscle potentiation does not account for adaptation in distance perception based on oculomotor cues. Perception \& Psychophysics, 1977, 22, 427-430.

Welch, R. B. Perceptual modification: Adapting to altered sensory environments, New York: Academic Press, 1978.

\section{NOTES}

1. Data are relatively scarce, but available decay studies suggest that, except for the dissipation of small induced effects, spontaneous decay in the dark is insufficient to reduce aftereffects to preexposure levels (Ebenholtz, 1981; Ebenholtz \& Wolfson, 1975; Paap \& Ebenholtz, 1977).

2. The slight increase in phoria aftereffect may reflect the fact that accommodation was somewhat relaxed and not fully maintained for the target distance of $20 \mathrm{~cm}$.

3. This was in excellent agreement with the slope of $1.35 \mathrm{ob}-$ tained from the calibration function of a previous study (Ebenholtz, 1981). 
4. Calculations of convergence angle were based on the average interocular axis of $6.07 \mathrm{~cm}$.

5. The slope of .26 is smaller than the $.6-.7$ range found previously (Ebenholtz, 1981). These differences in gain may reflect characteristics of the target pattern, such as its spatial frequency, that modulate its capacity to stimulate accommodation during the manual pointing procedure (Charman \& Tucker, 1977).

6. The post-pre differences in pointing were weighted by a factor determined by taking the difference in preexposure pointing scores between the target at $33.33 \mathrm{~cm}$ and one at $38.5 \mathrm{~cm}$ and dividing by the actual distance between these targets. This ratio was calculated for each of the four sessions and averaged separately for each subject. The inverse of the averaged ratio was then used as the desired individual weighting factor. These ranged from .63 to 2.13.

7. Dark vergence represents the position of physiological rest of the vergence system as measured in the absence of any accom- modative stimulus. Although it probably is highly correlated with conventional measures of phoria, there are no data on this point. 8. Another reason to expect differences in adaptation lies in the failure of Wallach and Halperin (1977) to equate the groups for oculomotor activity during the adaptation period. Paap and Ebenholtz (1977) demonstrated that distance aftereffects can be completely eliminated by horizontal (version) eye movements of the same type required by Wallach and Halperin of the group that failed to adapt. Yet another possible reason for unequal adaptation effects in Wallach and Halperin (1977) is associated with the fact that their groups were not equated for lateral head movements. Since it is not known what effect there is of the vestibular ocular reflex on accommodation, it would seem to be appropriate to equate head movements in the two conditions.

(Manuscript received September 4, 1981; revision accepted for publication February 26, 1982.) 